\title{
MINORÍAS Y MAYORÍAS SEXUALES: UNA TAREA PENDIENTE PARA LA PSICOLOGÍA Y LA EDUCACIÓN FORMAL EN EL PERÚ*
}

\author{
RICARDO BRAUN \\ https://orcid.org/0000-0002-0547-9618 \\ Universidad de Lima \\ Correo electrónico: rbraun@ulima.edu.pe
}

\author{
Recibido: 31 de mayo del 2021 / Aceptado: 14 de junio del 2021 \\ doi: https://doi.org/10.26439/persona2021.n024(1).5313
}

\begin{abstract}
Resumen. Este artículo tiene por finalidad examinar la situación de la educación escolar con respecto a la inclusión de las minorías sexuales dentro del currículo formal. Como no existen, la psicología, como ciencia de la conducta humana, tiene el rol de intervención a nivel escolar. Dado que el conocimiento acerca de la sexualidad proviene de una psicología popular y dado que los maestros escolares revelan desconocimiento científico acerca de la sexualidad en general, los profesionales de psicología que laboran en centros escolares en el Perú tienen la tarea pendiente de instruir a los docentes que se encargarán de la implementación de los lineamientos de la educación sexual integral emitida por el Ministerio de Educación del Perú. Se analizan los conceptos de psicología popular y psicología científica. Y se propone un tercer paradigma acerca del afrontamiento de los adolescentes de la minoría sexual, que no reemplaza, sino complementa los dos anteriores, el paradigma del contexto de la minoría sexual.
\end{abstract}

Palabras clave: minorías sexuales / adolescente escolar / educación sexual integral / Perú

\footnotetext{
*Agradezco al Programa de Estudios Generales de la Universidad de Lima por el auspicio para la investigación y preparación del artículo.
} 


\title{
SEXUAL MINORITIES AND MAJORITIES: A PENDING ASSIGNMENT FOR PSYCHOLOGY AND FORMAL EDUCATION IN PERU
}

\begin{abstract}
This essay examines the current situation of formal inclusion of sexual minorities in primary and secondary schools in Peru. There is no inclusion, therefore, psychology as a science has a pending task of intervening to overcome the situation. As the knowledge about sexuality comes from popular psychology and the teachers lack scientific knowledge about sexuality in general, and therefore, are not able to convey proper teaching to their pupils in charge, psychologists have the task to help teachers to implement the Ministry of Education of Peru's Integral Sexual Education program. I analyze the concept of folk psychology to better understand the difference between an intuitive explanation of sexuality versus an informed scientific one. I propose at the third paradigm in the analysis of adolescent sexual minorities That doesn't replace, but complements, the former ones, the paradigm of the context of the sexual minority.
\end{abstract}

Keywords: sexual minorities / adolescents in school

/ integral sexual education / Peru 


\section{INTRODUCCIÓN}

Denomino minoría sexual al grupo cuya identidad sexual, orientación o conducta difiere de la mayoría numérica de la sociedad. Constituyen minoría, entonces, individuos que se autoidentifican como lesbianas, gays, bisexuales, transgénero y otras posibilidades. La mayoría sexual, en consecuencia, es constituida por individuos cuya identidad, orientación y conducta es predominantemente heterosexual.

Tenemos una tarea pendiente. Es una que requiere ser continuada tanto para uno mismo como para las demás personas. Las preguntas sobre nuestra conducta sexual, particularmente cuando no llegamos a comprender su cadena causal, causan experiencias de perplejidad, que originan respuestas intuitivas - usualmente vagas y parcialmente correctas, en el mejor de los casos, o explicaciones falsas- que parecieran ser el núcleo de la sabiduría popular con respecto a este complejo fenómeno humano. Nuestra natural curiosidad requiere ser contestada, pero en forma adecuada, que mejor refleje la realidad. Las personas con orientación heterosexual, evidentemente la mayoría numérica, pueden tener una concepción de las minorías sexuales fruto de su experiencia y no de la información adecuada que deberían de haber recibido durante su formación escolar. Sus pares en minoría no se distinguen en la adquisición del conocimiento. En eso son iguales.

Sin embargo, esta situación no tendría que ser tan relevante si no conllevara un costo emocional cuando se trata de nuestros estudiantes que se encuentran en una de las etapas de mayor impacto en su desarrollo sexual, la etapa escolar.

En este ensayo quiero demostrar la necesidad de que la ciencia de la psicología tome el papel protagónico en la instrucción y formación de padres y madres de familia, docentes y estudiantes de educación básica. Los datos acerca de la necesidad de información y la carencia de conocimientos acerca de la sexualidad tanto de docentes como escolares son preocupantes y la ciencia del bienestar humano no puede ser ajena ante esta realidad. En la primera sección describo brevemente los antecedentes acerca del estudio científico de la sexualidad permeada primordialmente por el psicoanálisis freudiano. En la segunda sección discuto la pertinente distinción entre la psicología popular y la psicología científica. Es esta última la fuente de la transmisión de conocimientos que se debe impartir en las escuelas, no la psicología cotidiana que resulta, en fenómenos complejos, falsa. En la tercera sección presento la situación de la educación sexual actual en el Perú, a partir de los esfuerzos que hizo el Ministerio de Educación del Perú, y argumento acerca de la necesidad de que la psicología científica tenga un rol preponderante en la tarea de la instrucción sexual, puesto que los conocimientos adquiridos a través del sentido común distan peligrosamente del conocimiento proveniente de la ciencia. En la cuarta sección se presentan los tres paradigmas que se utilizan para describir el afrontamiento de las personas de orientación no heterosexual, con el objetivo de demostrar 
que los tres no son excluyentes, sino necesarios para una mejor comprensión de la situación de la minoría sexual.

\section{EL ESTUDIO CIENTÍFICO DE LA SEXUALIDAD Y LA ORIENTACIÓN SEXUAL}

Freud es, sin duda, el personaje en la historia de la psicología que más impregna la cultura popular con respecto a la sexualidad. Su Psicopatología de la vida cotidiana (1960) lo elevó a la fama, si lo juzgamos a través de la influencia en la cantidad de expresiones, suposiciones y explicaciones que nos muestran los medios de comunicación masiva. Términos como "inconsciente", "complejo de Edipo", "libido", "símbolo fálico" están incluidos en la conversación informal de estudiantes y profesores universitarios. El cine tampoco es ajeno a la influencia de suposiciones ontológicas y procesos del desarrollo sexual, como el personaje Alvi en Annie Hall de Woody Allen: "Pues yo jamás tuve un periodo de latencia. No es culpa mía". Otras cintas como Volver al futuro y El Imperio contraataca aluden a la dinámica del complejo de Edipo, según S. Mariansky, del Museo Freud de Londres (Kelly, 2014). Si la obra de Freud es comparable a los efectos paradigmáticos de Copérnico y Darwin, seguramente continuará siendo tema de charla académica, que considero para pocos, hoy en día (Thagard, 2010; Weinert, 2009). Pero la "envidia del pene" como explicación del desarrollo de la sexualidad femenina no tiene lugar en la Academia contemporánea, salvo en la historia de la psicología.

Freud es más comentado y citado que Magnus Hirschfeld, el fundador del Institut für Sexualwissenschaft [Instituto de la ciencia del sexo], primero y único en su género. En él trabajaron cuarenta investigadores, y si bien no hay acceso a la información detallada, Hirschfeld representaba una mirada fresca, innovadora y progresista acerca de la concepción de la homosexualidad y otras orientaciones no heterosexuales. De hecho, él es creador del -ahora cuestionado- concepto del "tercer sexo". No sería justo evaluar a una persona de inicios del siglo xx con la ciencia estándar del 2021. Sin embargo, su actitud hacia el estudio de la sexualidad lo llevó a promover la despenalización de las personas de orientación homosexual. No tenemos los archivos de las investigaciones porque el nazismo se encargó de desaparecer la biblioteca e incendiarla por considerar que las investigaciones realizadas eran judías (Hirschfeld como Freud eran de ascendencia judía), socialdemócratas y ofensivas para la moral pública.

La desaparición del Instituto y de sus documentos fue un ataque al inicio del estudio científico de la sexualidad que luego de la Segunda Guerra Mundial estaría dominado por la influencia del psicoanálisis, particularmente en el Reino Unido y en Estados Unidos de América. 
Durante la segunda mitad del siglo xx la Asociación Americana de Psiquiatría estuvo formada mayormente por psicoanalistas, fruto de la inmigración de médicos de Europa Central que huían del nazismo y estalinismo. El DCM I y II determinaron, como era obvio por su persuasión teórica, que la homosexualidad era una enfermedad, de acuerdo al canon de deficiencia que caracterizara la concepción freudiana de la homosexualidad. Las otras formas de orientación sexual no existían. Pero, conforme se fue desmedicalizando la práctica psicoanalítica, los psiquiatras provenían de otras fuentes teóricas, y en 1980, la tercera edición del manual fue editada por un grupo de psiquiatras no analistas de la Universidad de Washington (St. Louis) (Menand, 2017), y al día siguiente de su publicación, amanecieron curadas todas las personas con orientación hacia el mismo sexo.

Si hago mención del psicoanálisis es porque el inicio del estudio científico de la diversidad sexual fue bajo la suposición de que se trataba de una enfermedad, desviación, problema o como quiera denominarse. Cualquier sinónimo o analogía, como "problema", remitía a una infracción, sea biológica o mental. Un ejercicio pueril demuestra mi punto. Cuando se busca explicación del origen causal de la heterosexualidad, Google no trae tanta información si acaso colocamos "etiología" en el explorador. Si intento con los siguientes términos: "etiología / heterosexualidad", serán pocas las correspondencias para "heterosexualidad". La respuesta del explorador, empezando con la segunda entrada, será dirigida a homosexualidad. Si queremos una búsqueda más exitosa, necesitamos consignar un término que no indique enfermedad: causa u origen.

No ocurre lo mismo si probamos la misma búsqueda con los términos "etiología / homosexualidad". Bingo. La etiología es el término usual que significa causa de una enfermedad o mal. Aun cuando no sea su significado etimológico, la costumbre y la práctica médica así los determinan. La segunda entrada los remitirá a homosexualidad. "Causa" satisface nuestra curiosidad explicativa, "etiología", nuestro diagnóstico clínico.

Felizmente, la ciencia psicológica tiene un cuerpo de conocimientos que desafía completamente la concepción que caricaturizaba Cattell.

La psicología científica ha contestado a una serie de interrogantes, como las preguntas que podemos hacernos acerca del arte. $Y$ respuestas con una explicación causal. Por ejemplo, ¿cuál es el tipo de arte que más emociona, pintura o música? Respuesta: la música, porque una pintura es observada por pocos minutos, mientras que la música es percibida por varios minutos y puede involucrar movimientos corporales, además. ¿Y más emoción que la música? Respuesta: el cine, que tiene imagen y sonido (Winner, 2019). La respuesta no es definitiva como la mayoría de respuestas en la ciencia, pero una respuesta, como otras que la psicología del siglo xx propone, son resultados de la investigación teórica y empírica. Lo que ocurre en psicología del arte es aplicable a las otras áreas, en nuestro caso, en la psicología del sexo. Tenemos una considerable cantidad de estudios recientes que, si bien se originan en países industrializados, 
podemos tomar como inspiración; algunos títulos, temáticas o preguntas, con la debida adaptación a nuestra realidad y culturas, pueden ser aplicados.

La diversidad de teorías y enfoques crea para el lego la sensación de precariedad e inmadurez. Pocas veces ven esa práctica como resultado de la complejidad del objeto de estudio. Para los que conocen y practican la disciplina, pueden ver esa diversidad como riqueza de la ciencia.

Pero la ciencia psicológica no es psicología popular.

II

\section{LA PSICOLOGÍA POPULAR Y LA PSICOLOGÍA CIENTÍFICA}

La cultura y la psicología populares no constituyen la guía científica para la comprensión de la realidad, y menos para conducir adecuadamente la práctica psicológica y la educación de estudiantes en la niñez y adolescencia. Dado que la psicología y la educación como ciencias y profesiones comparten el vocabulario proveniente del lenguaje ordinario, están más expuestas a la vaguedad, a la sobresimplificación y al equívoco, entre otros problemas presentados por los trabajos de Freud y otros desde sus inicios. En la conocida frase de Cattell se advierte esta preocupación: “Pero la psicología es una disciplina más complicada, en la que se ha sabido de destacadas autoridades que caen en círculos, describiendo cosas que todo el mundo sabe, en un lenguaje que nadie entiende" (Cattell, 1966, p. 18).

La diferencia entre la psicología popular y la psicología científica es saltante. La física popular se distingue de la física científica en mucho, desde la teoría atómica hasta la explicación de los fenómenos cotidianos. La botánica popular tiene respuestas como las que me contestan mis estudiantes: "El agua alimenta a las plantas" (desconocimiento del papel de disolvente de los minerales) y "La temperatura de evaporación del agua" (100 grados Celsius, aun cuando se les confronta con la temperatura del mar donde se bañan). Mutatis mutandis, se aplica para la psicología científica en contraste con la popular.

La formación y prácticas científicas pueden distinguirse de prácticas no científicas, en por lo menos dos características: la apertura a la incertidumbre (rechazo del dogmatismo) y la práctica basada en evidencias (PBE). Una persona formada como científica no solo aprende un cuerpo de conocimiento, sino también, mediante hábitos promovidos, una serie de actitudes. La enseñanza científica no puede ser dogmática, por más personas dogmáticas que existan dentro de la ciencia. Los ejemplos de actitudes dogmáticas son numerosos, pero ello no justifica su existencia. De hecho, la actitud dogmática solo retrasa la aceptación de las evidencias, como ocurrió en un caso paradigmático de la medicina reciente: la aceptación de la hipótesis del origen bacteriano de algunas 
úlceras pépticas tomó varias décadas, ante la hostil recepción del descubrimiento, desde 1982 hasta la entrega del Premio Nobel en el 2005. La nota de prensa de la Fundación Nobel explícitamente decía que "Barry Marshall y Robin Warren... con tenacidad y mente preparada, desafiaron los dogmas prevalentes" (The Nobel Foundation, 2005).

La historia de Marshall y Warren es instructiva en la medida que nos acerca a las prácticas reales de la actividad científica. No es una empresa romántica desprovista de prejuicios e intolerancia. Pero la prevalencia de la aceptación de la incertidumbre y la PBE siguen siendo nuestras mejores herramientas intelectuales para el éxito de esta empresa. En efecto, Kamhi enfatiza que la persona formada en la actitud científica crea un sistema de control interno de tal modo que ante la incertidumbre recurre a la práctica que ya ha asimilado durante su formación: verificación externa y la revisión por pares del discurso científico (Kamhi, 2011). Todo estudiante científico tiene que entender y valorar nuestros sistemas públicos formales de confianza: congresos y publicaciones serias.

La formación y prácticas científicas tienen un ethos compartido que se enseña en la Academia y se ejerce en la profesión. Pero no puede ser el mismo desde Hipócrates - Arquímedes hasta nuestros días. La ortodoxia puede tener un valor temporal, pero la historia de la ciencia es una de permanencia y cambio. El progreso en teoría y práctica no requiere evidencia, pero se basa en la misma respuesta ante la incertidumbre que señalé arriba. La confrontación de ideas produce la necesaria dinámica. Esa dinámica la ilustra correctamente Kuhn (1977) como la "tensión esencial" entre la "convergencia" y "divergencia". Los estudiantes son iniciados en un modelo vigente y se les enseña a converger con teorías, conceptos, ejemplos y conductas. Pero también son incentivados para adoptar la actitud escéptica, la desconfianza en el autoritarismo y dogma, y la posibilidad de tener un punto de vista discrepante. En suma, la divergencia. Los libros de texto son el mejor ejemplo de la convergencia, y las tesis doctorales y la investigación profesional, de la divergencia.

Y creo que el análisis de Kuhn describe adecuadamente la situación en el tema que abordo en este ensayo. Los nuevos paradigmas acerca de la diversidad sexual han surgido de la incertidumbre explicativa y predictiva que generaron modelos anteriores. Sin embargo, a diferencia de la clásica visión kuhniana de la ciencia, paradigma vigente-anomalías-crisis-revolución científica-nuevo paradigma, no siempre existe un reemplazo total. Pueden sobrevivir paradigmas diferentes para explicar. En una población cualquiera encontraremos que los componentes y características son tan diferentes que un solo paradigma no es capaz de explicarlo todo. El mejor ejemplo viene del mismo campo al que, limitadamente, Kuhn recurría para las ilustraciones: la física. A más de trescientos años de la publicación de los Principia de Newton, cualquier vuelo comercial seguirá utilizando las suposiciones y ecuaciones que son parte de ese manual clásico. Para otros fenómenos de alta velocidad, Einstein reinará. Esto vale más para el problema 
de la conducta sexual humana, cuya diversidad puede desafiar un solo paradigma explicativo y normativo.

La psicología científica tiene respuestas que permiten orientar nuestro trabajo en las diferentes áreas de competencia profesional. En el caso del análisis de la diversidad sexual, tenemos la suficiente bibliografía de investigación que permite elaborar juicios basados en la realidad empírica y no la fantasía imaginativa o anecdotario.

Veamos cómo podemos intervenir ante la penosa situación en el Perú.

III

\section{LA EDUCACIÓN SEXUAL ESCOLAR EN EL PERÚ}

En el 2008 se actualizaron los lineamientos de la Educación Sexual Integral (ESI), en el 2016 se integraron al Currículo Nacional de Educación Básica, y a la fecha no se ejecutan plenamente. Si el Ministerio de Educación (Minedu) muestra preocupación por la mayoría, la minoría, simplemente, no existe.

Es ese vacío que la psicología está llamada a llenar. La ciencia tiene el lugar que le corresponde para completar lo que parece que nos tiene indolentes. Cuando se redactó el documento de la ESI, hubo un estudio preliminar que arrojó estadísticas alarmantes, que obviamente se trataron de hacer invisibles con aquella campaña nefasta contra la denominada "ideología de género". Como si la ideología en contra de la ideología de género no fuera otra ideología.

Técnicamente (según se ha documentado) el término es utilizado por primera vez por Destutt de Tracy, filósofo de la Revolución francesa, para designar a su "ciencia de las ideas" cuya finalidad era servir a la gente, inclusive salvarla, librándola de los prejuicios y preparándola para el reinado de la razón. Pero, curiosamente, desde su inclusión, el término ha sido usado en forma laudatoria o despectiva. El uso peyorativo del término puede ser rastreado en Marx y otros. Pero, esencialmente, no tiene por qué tener un uso negativo. De manera que podemos tener ideología democrática, cristiana, liberal, conservadora, etcétera.

Las estadísticas mencionadas señalan que, por parte de los docentes, "60 \% de tutores sienten que no están capacitados para abordar la educación sexual, que no se sienten cómodos al hablar del tema, no saben cómo tratarlo con los estudiantes de diversas edades, les da vergüenza, se sienten inseguros. Hay otros que no están convencidos de que deba impartirse la educación sexual" (Minedu, 2008). Y en el caso de los estudiantes, reporta el documento del Minedu,

el 84 \% de escolares de secundaria de la Educación Básica Regular de Lima y provincias informan que sus tutores han abordado el tema de educación sexual, 
sobre todo en lo referente a la prevención de las ITS, el VIH-SIDA y el embarazo adolescente ( $23 \%$ ) y a los derechos sexuales y reproductivos (21\%), y menos sobre otras dimensiones, como el respeto y la responsabilidad para con la pareja, el cuidado del cuerpo o los planes de vida. (Minedu, 2008)

Es claro que el enfoque de la educación sexual escolar sigue siendo médico y no de bienestar. Y de diversidad, una tímida mención, en el marco de los objetivos educacionales en el tema de derechos humanos y orientación sexual: "Examinar críticamente actitudes acerca de la orientación sexual, en el marco de los derechos de las personas" (Minedu, 2008). Es todo. Y ese objetivo está consignado en el VII ciclo de educación secundaria. Suficiente. La otra mención del término "diversidad" se redacta con cierta elaboración, pero se desvía el tema hacia la diversidad cultural, que ciertamente no es despreciable, pero no es tan relevante para focalizar en el tema de la diversidad sexual, particularmente en un manual que pretende dar respuestas al declarado $60 \%$ de encuestados.

Los estudiantes (más de la mitad de los encuestados) también revelaron que preferían conversar acerca de la sexualidad con sus pares. Nos imaginamos que, gracias a internet, pueden obtener conocimientos de diversas fuentes. Pero internet ofrece información con y sin rigor. Puede iluminarnos o confundirnos o engañarnos. Si no tuviéramos alternativa, sería nuestra única fuente. Pero la alternativa existe y está en las escuelas: los profesionales de la psicología que laboran en las escuelas.

IV

\section{UN NUEVO PARADIGMA COMPLEMENTARIO}

Como arriba indiqué, es posible trabajar con diferentes paradigmas dependiendo de la necesidad explicativa y predictiva. En el estudio de la diversidad sexual hemos sido adiestrados en el pasado con el paradigma del déficit, uno inspirado en la tradición freudiana que describí. En este paradigma (Martin y Hetrick, 1988), que consideraba que las minorías sexuales (el término no es el original, pero lo estoy modificando para adaptarlo a la terminología que empleo) eran víctimas de los servicios de salud, sufrían abusos verbales físicos y mentales en las escuelas, tenían problemas de desempeño académico y constituían una población potencialmente suicida. De manera que el paradigma asociado con "minoría en riesgo" se ha ido modificando a otro paradigma que no resalta el clásico modelo de déficit. El segundo paradigma, que fue basado en numerosas entrevistas a profundidad, reemplaza el concepto de déficit por el de "resiliencia". Savin-Williams y otros encontraron que los individuos de estas minorías no querían ser clasificados como víctimas, ni se sentían en riesgo constantemente. Que mientras advertían que su orientación les hacía más vulnerables, y tanto más cuanto más fueran visibles, también reportaban que eran capaces de confrontar sus dificultades y superarlas con éxito (Savin-Williams, 2005). 
Sin embargo, estos paradigmas tienen un elemento común. Tratan a las minorías sexuales como si fueran un grupo monolítico u homogéneo. Lo mismo se aplica para las mayorías sexuales. Sin embargo, las historias personales distan mucho de ser semejantes, no solo si comparamos variables como localización urbana o rural, sino que dentro de cualquier grupo de comparación aparecen diferencias que no pueden ser desconocidas. El nuevo modelo quiere ir más allá de los dos anteriores. Se concentra en la comprensión de las maneras como los adolescentes de las minorías sexuales negocian su desarrollo en el seno de sus variados contextos sociales (Horn, Kosciw y Russell, 2009). El componente adicional a los anteriores paradigmas es el contexto. Quiero enfatizar que no es necesario hacer un cambio paradigmático en nuestras investigaciones y prácticas profesionales, sino poder movernos en uno y otro paradigma de acuerdo a la situación individual de las personas con las que trabajamos y queremos asistir. Algunos adolescentes pueden estar en situación de riesgo, mientras que otros pueden ser resilientes. Nuestra intervención tiene que ser como sastre, a la medida. Los autores de este paradigma sostienen que es más enriquecedor estudiar las formas en que, por ejemplo, un ambiente hostil como la escuela se junta con un ambiente de soporte en la familia. Puede ser que la escuela sea particularmente agresiva, cuando se escuchan mensajes que violentan a las minorías. Sin embargo, podría encontrarse una adolescente a quien ese entorno no le afecta porque tiene un apoyo de su iglesia local, que le proporciona un rol protector. Así, encontrando los posibles protectores sociales, se pueden diseñar mejores instrumentos de intervención para la consejería. El paradigma del contexto ofrece a la ciencia y profesión psicológica una nueva forma de enfrentar un viejo problema.

La comprensión de uno mismo es un problema en sí mismo, que afecta tanto a las minorías como a las mayorías. Nuevamente insisto en el error de considerar a la población adolescente como un grupo homogéneo. Por la natural tendencia a la homogeneización, nos equivocamos al taxonomizar y querer que la talla única sea nuestra guía. Tómese el ejemplo de la menstruación. La menarquia ha variado sustancialmente desde el siglo XIX, pero tampoco es homogénea. Para muchas niñas puede ser un momento de alegría o de confusión. Pero no ayuda entrar a clase y hacer suposiciones basadas en la asociación edad-menarquia y dar consejos que no se aplican a todas.

Lo mismo ocurre con la identidad sexual. Pero puede ser más angustiante con la identidad de ser parte de una minoría. El desarrollo de la sexualidad, en particular de la minoría, es un proceso que requiere mucho cuidado al momento de taxonomizar. No hay hitos en el desarrollo sexual. Una niña con orientación homosexual puede no querer aceptarla y demorará en sentirse cómoda con sus deseos. Precisamente, Savin-Williams (2005), dialogando con adolescentes universitarios, encontró que si bien eran conscientes de su homoerotismo, no querían ser identificados por los demás como lesbianas o gays. Es más, no querían ser clasificados. Punto. La normativa que se puede señalar para esta 
realidad es que todo maestro escolar debe orientar hacia el bienestar y la aceptación personal. Pero eso no se logrará, en muchos casos, en el periodo escolar. Los estudios señalan que, durante el periodo escolar son pocas las personas que podrán sentirse cómodas con su orientación sexual minoritaria (Canadian Paedriatic Society, 2008).

La identidad sexual se va construyendo y en la escuela se dan los primeros pasos, no la graduación. Las maestras y maestros, junto con la ciencia psicológica, pueden ayudar a construirla en forma sana y feliz. No es tan difícil. Simplemente hay que hacer aparecer a esta minoría olvidada. No es magia. Están en nuestras aulas.

\section{CONCLUSIÓN}

En suma, no se puede continuar con esta situación. Las minorías sexuales en la escuela no existen, al menos en el discurso formal y en la literatura pertinente que emana del Ministerio de Educación. La ciencia psicológica tiene las herramientas intelectuales y prácticas para intervenir adecuadamente, puesto que no puede dejar en manos de la psicología popular el acompañamiento para las minorías sexuales en la escuela. Además de los dos paradigmas que se han usado para la comprensión de las minorías sexuales adolescentes, ha surgido un tercero que incorpora el contexto como un elemento sustancial en el análisis de las negociaciones que tiene que hacer para mejor adaptarse al entorno. Es importante recordar que el estudio de las minorías sexuales no es un estudio a un grupo homogéneo, puesto que no todos están en riesgo ni todos son resilientes. Tenemos que indagar en un contexto que permita comprender mejor los roles protectores que pueden tener diversos factores sociales, como la familia, los amigos, el grupo religioso y otros. Esta es una tarea pendiente que no puede esperar. Las minorías sexuales escolares han estado silenciadas por demasiado tiempo en el Perú.

\section{REFERENCIAS}

Canadian Paedriatic Society. (2008). Adolescent Sexual Orientation. Paedriatic Child Health, 13(7), 619-623.

Cattell, R. (1966). The Scientific Analysis of Personality. Aldine Publishing Company.

Horn, S., Kosciw, J., y Russell, S. (2009). New Research on Lesbian, Gay, Bisexual, and Transgender Youth: Studying Lives in Context. Journal of Youth Adolescence, 38, 863-866.

Kamhi, A. G. (2011). Balancing Certainty and Uncertainty in Clinical Practice. Language, Speech, and Hearing Services in Schools, 42, 59-64.

Kelly, J. (2014). Sigmund Freud: The Phrases You Use Without Realising It. BBC News Magazine. https://www.bbc.com/news/magazine-29251040 
Kuhn, T. (1977). The Essential Tension Selected Studies in Scientific Tradition and Change. The University of Chicago Press.

Martin, A. D., y Hetrick, E. S. (1988). The Stigmatization of the Gay and Lesbian Adolescent. Journal of Homosexuality 1, 163-183.

Menand, L. (21 de agosto del 2017). Why Freud Survives. The New Yorker. https://www. newyorker.com/magazine/2017/08/28/

Ministerio de Educación. (2008). Lineamientos educativos y orientaciones pedagógicas para la educación sexual integral. Manual para profesores y tutores de Educación Básica Regular. http://bvs.minsa.gob.pe/local/minsa/1283_gob523.pdf

Savin-Williams, R. C. (2005). The New Gay Teenager. Harvard University Press.

Freud, S. (1960). The Standard Edition of the Complete Psychological Works of Sigmund Freud (vol. VI). The Hogarth Press and the Institute of Psychoanalysis.

Thagard,P.(2010).Scientific Revolutions and ScienceEducation.ScienceandEducation, 20(9), 2109-2139

The Nobel Foundation. (2005). Press release. The Nobel Prize in Physiology or Medicine 2005. https://www.nobelprize.org/prizes/medicine/2005/press-release/

Weinert, F. (2009) Copernicus, Darwin, and Freud: Revolutions in the History and Philosophy of Science. Wiley-Blackwell.

Winner, E. (2019). How Art Works: A Psychological Exploration. Oxford University Press. 